\title{
Effects of Osteopathic Manipulative Treatment on Sleep Quality in Student Athletes After Concussion: A Pilot Study
}

Samantha Mazzeo, OMS III; Craig Silverberg, OMS III; Tiffany Oommen, OMS III; Diane Moya, OMS II; Nicole Angelo, OMS IV; Hallie Zwibel, DO; Jayme Mancini, DO, PhD; Adena Leder, DO; Sheldon C. Yao, DO

From the Departments of Family Medicine (Dr Zwibel) and Osteopathic Manipulative Medicine (Drs Mancini, Leder, and Yao) at the New York Institute of Technology College of Osteopathic Medicine (Student Doctors Mazzeo, Silverberg, Oommen,

Moya, and Angelo) in Old Westbury, New York.

Financial Disclosures: None reported.

Support: None reported.

Address correspondence to

Sheldon C. Yao, DO,

New York Institute of Technology College of Osteopathic Medicine, Department of Osteopathic Manipulative Medicine, PO Box 8000 Northern Blvd, Serota Building Room 127, Old Westbury, NY $11568-8000$.

Email: sheldon.yao@nyit.edu

Submitted December 14, 2018; revision received January 14, 2020; accepted February 20, 2020.
Context: Impaired sleep quality is among many symptoms observed in patients with a concussion and may predispose a patient to a prolonged recovery course and a later return to their daily activities. Studies have suggested that osteopathic manipulative treatment (OMT) may play a role in improving sleep quality.

Objective: To investigate how OMT may play a role in the management and overall healing process in patients with a concussion by improving sleep quality.

Methods: Data were collected from a randomized, controlled study on OMT and concussion (of which this study represents $1 \mathrm{arm}$ ) to investigate the effects of OMT vs concussion education counseling on sleep quality in student athletes with a concussion. Student athletes with no medical history of neurodegenerative disease who presented to the New York Institute of Technology College of Osteopathic Medicine Academic Healthcare Center with a concussion following a sport-related injury were enrolled in the study. Participants received OMT intervention or standard counseling on how to care for a concussion during their first and second visits. Participants rated their symptoms, including sleep quality, on the validated scale Sport Concussion Assessment Tool 5th Edition at 3 consecutive visits during 1 week. The mean sleep quality score within and between the OMT and education groups before each of 2 interventions and at the third visit were compared and analyzed using the Mann-Whitney $U$ test.

Results: Thirty participants were enrolled in the study. Total symptom data showed a stronger, significant correlation with sleep scores than with other symptoms. Participants receiving OMT $(\mathrm{n}=16)$ reported overall $80 \%$ and $76 \%$ improvement in sleep quality from pre-OMT values to their second and third visits, respectively. Participants who had an educational intervention $(n=14)$ reported a $36 \%$ and $46 \%$ improvement from pre-OMT values to their second and third visits, respectively.

Conclusion: The beneficial relationship trend between OMT and sleep quality in patients with a concussion was not statistically significant. Owing to the limitations of this study, further research with a larger population and sham control participants is warranted. (Clinicaltrials.gov No. NCT02750566)

J Am Osteopath Assoc. 2020;120(9):615-622. Published online August 11, 2020 doi:10.7556/jaoa.2020.100

Keywords: concussion, insomnia, OMT, osteopathic manipulation, osteopathic medicine, sleep 


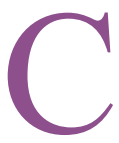

oncussions are a type of mild traumatic brain injury (TBI) in which a force to the head results in somatic, cognitive, and emotional symptoms. ${ }^{1}$ Between 2006 and 2010, the Centers for Disease Control and Prevention (CDC) reported approximately 1.8 million TBI-related emergency department visits. ${ }^{2}$ The CDC estimates that approximately 2.5 million concussions occur in the United States each year due to sports or physical activity; however, this number may be underestimated because these numbers were derived from emergency department visits and athletic training reports only. ${ }^{3}$ Currently, there is limited research investigating the relationship between concussions and resulting longterm sequelae.

Common physical symptoms of concussion include headache, nausea, vomiting, difficulty with balance, dizziness, changes in vision, and sensitivity to light or noise. Cognitive issues may arise, such as difficulty with attention and focusing, mental fogginess, and speech difficulties. ${ }^{1,4-6}$ Patients with a concussion may also experience emotional impairments such as irritability and emotional lability, as well as sleep-wake disturbances, such as excessive daytime sleepiness, sleep fragmentation, and insomnia., ${ }^{1,4-7}$

The cause of sleep disturbance in concussion may be multifactorial, including posttraumatic stress, pain, and inflammation from injury, or disruption of the sleepwake regulatory nuclei and pathways of the brain. ${ }^{8-10}$ Research has suggested that adequate, high-quality sleep with limited deprivation is critical for cognitive function. ${ }^{11,12}$ In addition, patients with concussion who have sleep disturbances experience a longer recovery period compared with patients with concussion without sleep disturbances. ${ }^{13}$ Thus, adequate sleep with minimal interruption may be an important factor for the concussion healing process, resolution of symptoms, and decreased incidence of long-term sequelae.

Several osteopathic manipulative treatment (OMT) techniques are used to treat a variety of bodily dysfunctions. In the current issue of The Journal of the American Osteopathic Association, Schwartzberg et $\mathrm{al}^{14}$ present data regarding somatic dysfunctions found in patients with concussion, where dysfunctions of the sphenobasilar symphysis have been found to be related to the direction of concussive force. Results from a separate arm of the clinical trial reported in the current study, in which we analyzed the efficacy of OMT vs concussion education on symptom severity, are also presented in this issue. ${ }^{15}$ Osteopathic cranial manipulative medicine is a technique used to treat dysfunctions of the cranium. ${ }^{9,16}$ Compression of the fourth ventricle (CV4) is a technique used to enhance motion of tissue and fluids, ${ }^{17}$ whereas venous sinus drainage (VSD) aims to minimize congestion by enhancing dural venous blood flow drainage from the skull to the main circulatory system. ${ }^{18}$ Research has demonstrated improvement in hemodynamic perfusion at the cranial base as measured by ultrasonographic encephalogram in patients receiving VSD compared with baseline and control groups. ${ }^{19}$ In addition, CV4 was previously found to significantly decrease sleep latency scores on multiple sleep latency testing and lower tone on muscle sympathetic nerve activity microneurography in healthy patients compared with placebo and no-treatment groups. ${ }^{20}$ The mechanism for decreased sleep latency after cranial manipulation is unknown.

Clinical assessments of cognitive impairment, physical signs and symptoms, neurobehavioral complaints, and sleep disturbances are some of the criteria used to diagnose concussions. ${ }^{1}$ A useful tool in identifying and monitoring symptoms is the Sport Concussion Assessment Tool 5th edition (SCAT5), a standardized neuropsychological test that subjectively measures the severity of concussion symptoms in athletes older than 13 years. $^{21}$ The evaluation includes indications for emergency referral, Glasgow Coma Scale, symptom severity scores, and physical and cognitive assessments. ${ }^{21}$ The purpose of this study was to investigate which SCAT5 symptoms are most affected by OMT compared with concussion education counseling (CEC) in student athletes with a concussion for future clinical decision making. "Trouble falling asleep" is 1 of 22 symptoms rated and was the category of focus for this study. 
We hypothesized that OMT would have a positive effect on sleep quality in participants with concussion. We anticipated that participants receiving OMT would have a more notable improvement in their sleep quality and thus an overall lower trouble falling asleep SCAT5 severity score among consecutive office visits, compared with participants receiving standard CEC.

\section{Methods}

\section{Recruitment}

This pilot study was a detailed analysis of data collected from a randomized, controlled trial of OMT in acute concussion approved by the New York Institute of Technology (NYIT) institutional review board and registered at clinicaltrials.gov (NCT02750566). Methods are described elsewhere in this issue, in the article summarizing a separate arm of our study. ${ }^{16}$ This study was conducted at NYIT College of Osteopathic Medicine Academic Healthcare Center (AHCC) in Old Westbury. Patients for recruitment were identified by physicians in the AHCC, including but not limited to referrals to the NYIT Sports Medicine Center, current patients, or student athletes from NYIT or surrounding universities. Diagnosis of concussion due to a sports injury was performed by board-certified physicians. After diagnosis, patients were notified of the current study and invited to participate.

Patients' eligibility criteria included presenting to the AHCC with a sports-related head injury within the past 2 weeks and concussion symptoms. Patients were excluded if they had contraindications to OMT or the inability to complete the assessment tools. Additionally, any patients who were pregnant or who had a past or present diagnosis of neurodegenerative disease were excluded from this study because these conditions could confound the data. For the safety of patients, any presentation suggesting a potentially lifethreatening injury were not included in this study and instead were advised to be evaluated in an emergency department. After inclusion and exclusion criteria were confirmed and informed consent obtained, participants were enrolled, randomized, and immediately attended their first visit.

\section{Participant Groups}

Participants were randomized into 2 treatment groups by block randomization. The OMT group received OMT techniques, and the CEC group received CEC only (controls). Participants in the OMT group received an osteopathic structural examination, an OMT session that addressed concussion-related somatic dysfunctions, and documentation of somatic dysfunctions per the guidelines listed in the Table. ${ }^{9,14}$ Examination and OMT of participants in the OMT group were performed by board-certified neuromuscular medicine/osteopathic manipulative medicine (NMM/OMM) or family medicine/OMT physicians. All 3 treating physicians (J.M., S.Y.) underwent training to ensure that key OMT techniques to address dysfunctions of the cranium, spine, rib cage, pelvis, and sacrum were used. These techniques included occipitoatlantal decompression, VSD, cranial lifts, CV4, thoracic inlet release, rib-raising, and abdominal diaphragm doming. Any additional severe and pertinent dysfunctions were managed with OMT. When possible, the same physician treated the same participant throughout their 3 visits. Dialogue between physician and participant in the OMT group regarding basic care for concussion was limited. Participants in the CEC group received extensive standardized CEC reviewing what to expect after a concussion, facts about concussion and brain injury, and how to manage symptoms at home, per the CDC and the American Academy of Family Physicians (AAFP) guidelines. Evaluation of participants in the CEC group was performed by a board-certified family medicine physician (H.Z.) or a board-certified neurologist (A.L.). Both groups received 30 minutes of face-to-face interaction with a physician.

\section{Data Collection}

Participants had a total of 3 consecutive visits. Visit 1 represented the initial visit when participants were first 
Table.

Guidelines for Osteopathic Structural Examination and Treatment for Participants Receiving Intervention

\begin{tabular}{lll} 
& Osteopathic structural examination & Osteopathic manipulative treatment \\
\hline Cranium & $\begin{array}{l}\text { Assessment of the OA joint, OM suture, strain } \\
\text { patterns, and cranial rhythmic impulse }\end{array}$ & $\begin{array}{l}\text { OA decompression, V-spread, venous sinus drainage, } \\
\text { BMT for strain patterns, cranial lifts; cranial treatment ends } \\
\text { with CV4 technique }\end{array}$ \\
\hline Spine & $\begin{array}{l}\text { Assessment for cervical, thoracic, and lumbar } \\
\text { somatic dysfunctions }\end{array}$ & $\begin{array}{l}\text { BLT, MET, FPR, articulatory techniques, HVLA, and } \\
\text { counterstrain }\end{array}$ \\
\hline Rib cage & $\begin{array}{l}\text { Assessment of first rib, thoracic outlet, sternal/ } \\
\text { clavicle dysfunction, thoracic diaphragm, and rib } \\
\text { 2-12 dysfunctions }\end{array}$ & $\begin{array}{l}\text { Thoracic outlet release, rib raising, and articulatory } \\
\text { techniques to address any rib cage dysfunctions }\end{array}$ \\
\hline Sacrum/ & Assessment of any somatic dysfunctions & Standard OMT to address any dysfunction \\
\hline
\end{tabular}

Abbreviations: BLT, balanced ligamentous tension; BMT, balanced membranous tension; CV4, compression of the fourth ventricle; FPR, facilitated positional release; HVLA, high-velocity low-amplitude; MET, muscle energy technique; OA, occipitoatlantal; OM, occipitomastoid; OMT, osteopathic manipulative treatment.

enrolled, evaluated, and received the first intervention. Visit 2 followed visit 1 by 48 to 72 hours, and visit 3 occurred approximately 1 week after visit 1 . At each visit, before any intervention, symptoms were assessed with the SCAT5. Participants completed the evaluation by providing a severity score, ranging from 0 to 6 , for each symptom. OMT and CEC were performed during visits 1 and 2 only. Visit 3 was scheduled for data collection only.

\section{Statistical Analysis}

Correlation of the SCAT5 scores with the "trouble falling asleep" SCAT-5 item between visits 1, 2, and 3 , were analyzed by linear regression. Group differences of the mean "trouble falling asleep" SCAT5 scores between visits 1 and 2 and visits 1 and 3 were analyzed using the Mann-Whitney $U$ test.

\section{Results}

Thirty participants (18 men [60\%] and 12 women [40\%]; mean age, 19.7 years) were enrolled in the study. The OMT group had 8 men and 6 women, and the CEC group had 10 men and 6 women. There was a significant, medium-strength correlation of the "trouble falling asleep" item with the total symptom score for all participants after injury $\left(Y=7.233 \mathrm{x}+22.278 ; r^{2}=0.302\right.$; $P=.001)$. There were large, significant correlations for visit $2\left(Y=12.673 \mathrm{x}+8.819 ; r^{2}=0.645 ; P<.001\right)$ and visit $3\left(Y=8.956 \mathrm{x}+5.268 ; r^{2}=0.471 ; P<.001\right)$. There were stronger correlations of the total symptom scores with the "trouble falling asleep" item than with the other symptoms.

On visits 1, 2, and 3, the mean SCAT5 scores for trouble falling asleep were $1.00,0.64$, and 0.54 , respectively, in the CEC group and 1.33, 0.27, and 0.36 , respectively, in the OMT group (Figure). The OMT group displayed an $80 \%$ improvement in sleep symptom severity from visit 1 to visit 2 , whereas the CEC control group displayed a 36\% improvement $(P=.24)$. When comparing visit 3 with the initial visit, the OMT group displayed a $73 \%$ improvement in sleep symptom score, and the CEC group showed a $46 \%$ improvement $(P=.24)$.

When comparing groups, analysis did not reveal statistical significance. 


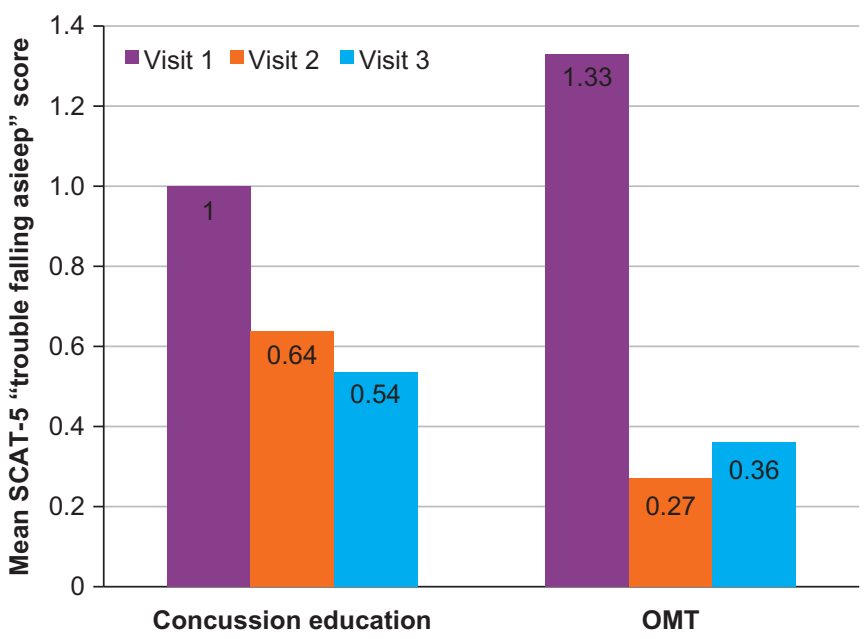

Figure.

Mean symptom ratings of "Trouble Falling Asleep" were calculated for each of the 3 visits and compared between the concussion education group and OMT group. "Trouble Falling Asleep" is 1 of the various symptoms rated on the SCAT- 5 survey (with 0 representing no symptoms and 6 representing severe symptoms). Abbreviations: OMT, osteopathic manipulative treatment; SCAT-5, Sport Concussion Assessment Tool fifth edition.

\section{Discussion}

We observed a trend toward improvement in sleep quality in both groups when comparing visits 1 and 2, as well as visits 1 and 3. The OMT intervention group displayed a relatively larger improvement in sleep compared to the CEC control group between visits 1 and 2 and visits 1 and 3. Despite a lack of statistical significance, valid clinical improvement may have been present, indicating that the use of OMT in patients with concussion may have helped, although no conclusions can definitively be drawn.

Lacrosse was the most common sport that caused concussion among our participants. Other epidemiologic reports have shown that football and soccer are among sports most often associated with concussion. ${ }^{22,23}$ Further studies on concussion in sports may investigate potential relationships between the mechanism of injury, osteopathic structural examination findings, and presenting symptoms among different sports.

This study used 1 item from the SCAT5 survey as a quantifiable, participant-rated measure of sleep quality. Changes in single items from the full SCAT5 survey after OMT were not studied separately. Subsequent studies may consider the use of more advanced measurements to increase the precision and accuracy of sleep outcomes. This may include validated sleep measurements (Pittsburgh Sleep Quality Index or the Epworth Sleepiness Scale), as well as objective physiologic measurements (actigraphy and polysomnography), which may assess detailed sleep parameters, including stratification of phases of sleep that may be affected by a concussion.

In otherwise healthy patients, sleep-wake cycle disturbances and sleep latency can be affected by several factors. Nonmodifiable risks include sex and age; modifiable factors include anxiety, depression, insomnia, acute or chronic pain, sleep apnea, and thyroid disease. ${ }^{11}$ In addition, behavioral choices such as caffeine intake, alcohol use, exercise schedule, and academic or extracurricular obligations may affect the sleep-wake cycle. ${ }^{12}$ Subsequent studies should include an additional intervention group of sham treatment to control for the placebo effects of light, nonpurposeful touch on both healthy participants without a history of concussion matched with participants with a concussion of similar age, athletic 
involvement, academic workload, and caffeine consumption.

A systematic review of sleep medicine by Sarris and Byrne $^{24}$ displayed supportive evidence for tai chi, yoga, and acupressure as treatment for insomnia, whereas other studies have suggested that massage therapy leads to significant improvement in sleep quality in postmenopausal $^{25}$ and postpartum ${ }^{26}$ women with insomnia. In addition, patients experiencing insomnia while in a hospital were more willing to accept nonpharmacologic treatments, such as massage therapy, sleep hygiene, music, and relaxation techniques for their insomnia compared with benzodiazepines. ${ }^{27}$ Future treatment options for insomnia may expand to include OMT in the overall treatment of patients with primary insomnia or insomnia due to a medical condition such as concussion.

The pathophysiology of concussions is not well understood, although areas of the brain involved in regulating sleep-wake mechanisms are subject to diffuse axonal injury with concussion. ${ }^{28}$ One study ${ }^{29}$ hypothesized that trauma-induced metabolic changes that lead to neurotoxic byproducts, altered blood flow, and disturbances of intraneuronal metabolism are among the physiologic changes that may occur and present as classic symptoms. A relief from a concussion-induced decrease in tissue compliance may be a potential mechanism of action underlying the trend of improved sleep quality after OMT. In this theory, the decreased tissue compliance is a result of somatic dysfunctions causing musculoskeletal and dural restrictions. Based on previous studies, ${ }^{1,4-13}$ the OMT techniques used were expected to decrease mechanical restriction, improve blood and lymphatic flow, and improve sleep latency. OMT may expedite the process of excreting neurotoxic byproducts and regulating disrupted metabolism, as exemplified by the circulatory and metabolic models of OMT. ${ }^{9,16-18}$ In addition, muscle strain throughout the body may also be present as a result of injury and may contribute to the poor sleep quality reported by patients. The use of OMT to address musculoskeletal complaints pertaining to the biomechanical model may improve pain and contribute to improved sleep quality.

Studies have demonstrated the safety of OMT in the management of TBI, with no adverse effects reported. ${ }^{30,31}$ Due to the nature of such injury, we suspect that the head trauma seen in patients with concussion, which may cause initial cranial somatic dysfunctions leading to subsequent somatic dysfunctions throughout the rest of the body, may respond to OMT. Case reports have demonstrated instances in which concussive symptoms have resolved after OMT. ${ }^{32,33}$

In addition, other cases have shown improvement in somatic dysfunctions with consecutive OMT sessions in patients with severe acute TBI. Although limited research exists exploring the effect of OMT on concussion symptoms, a case report by Guersney et al displayed improvements in symptoms and the Sensory Organization Test in a 27 -year-old patient with a 3-day history of concussion symptoms after a snowboarding accident. ${ }^{33}$ Moreover, a preliminary study by Burm and Goedhart ${ }^{34}$ proposed that osteopathic cranial manipulative medicine improves balance in challenging physical situations in healthy professional football players.

We acknowledge our sample size as a limiting factor of this study; a larger sample size would provide additional data on clinical response to treatment. Additionally, future studies would benefit from the use of an active control group to further compare the effects of touch and intention to treat with OMT in patients with a concussion. Using a tool such as the Insomnia Severity Index, future research could gender block athletes randomized to OMT, control (usual care), or sham treatment with 1 month of follow-up. Additional studies investigating how OMT may help patients with concussion are warranted.

\section{Conclusion}

A multidisciplinary approach to concussion management can help quicken recovery, decrease the incidence of long-term sequelae, and reduce the need for 
pharmacologic intervention. Although not found to be statistically significant in this study, OMT may have a role as an adjunctive treatment option in treating sleep symptoms experienced by patients with concussion, although more extensive research is needed to assess the short- and long-term effects of OMT on concussion symptoms.

\section{Author Contributions}

All authors provided substantial contributions to conception and design, acquisition of data, or analysis and interpretation of data; Drs Mazzeo, Mancini, Yao, and Zwibel drafted the article or revised it critically for important intellectual content; all authors gave final approval of the version of the article to be published; and all authors agree to be accountable for all aspects of the work in ensuring that questions related to the accuracy or integrity of any part of the work are appropriately investigated and resolved.

\section{References}

1. McCrory $\mathrm{P}$, Meeuwisse $\mathrm{W}$, Dvořák J, et al. Consensus statement on concussion in sport-the 5th international conference on concussion in sport held in Berlin, October 2016. Br J Sports Med. 2017;51 (11):838-847. doi:10.1136/bjsports-2017-097699

2. Percent distributions of TBI-related emergency department visits by age group and injury mechanism - United States, 2006-2010. Centers for Disease Control and Prevention. website. https://www.cdc.gov/ traumaticbraininjury/data/dist_ed.html. Accessed October 2018.

3. DePadilla L, Miller GF, Jones SE, Peterson AB, Breiding MJ. Self-reported concussions from playing a sport or being physically active among high school students — United States, 2017. MMWR Morb Mortal Wkly Rep. 2018;67:682-685. doi:10.15585/mmwr. mm6724a3

4. Evans RW, Whitlow CT. Acute mild traumatic brain injury (concussion) in adults. UpToDate.com website. https://www.uptodate.com/contents/ acute-mild-traumatic-brain-injury-concussion-in-adults. Accessed October 2018.

5. Kelly JP, Rosenberg JH. Diagnosis and management of concussion in sports. Neurology. 1997;48(3):575-580. doi:10.1212/wnl.48.3.575

6. Zwibel H, Leder A, Yao S, Finn C. Concussion evaluation and management: an osteopathic perspective. J Am Osteopath Assoc. 2018;118(10):655. doi:10.7556/jaoa.2018.144

7. Lim MM, Baumann CR. Sleep-wake disorders in patients with traumatic brain injury. UpToDate.com website. https://www.uptodate. com/contents/sleep-wake-disorders-in-patients-with-traumatic-braininjury. Accessed October 2018.

8. Viola-Saltzman M, Watson NF. Traumatic brain injury and sleep disorders. Neurol Clin 2012; 30(4):1299-1312. doi:10.1016/j. ncl.2012.08.008

9. Hitscherich $\mathrm{K}$, Smith $\mathrm{K}$, Cuoco JA, et al. The glymphatic-lymphatic continuum: opportunities for osteopathic manipulative medicine. J Am Osteopath Assoc. 2016;116(3):170-177. doi:10.7556/jaoa.2016.033
10. Baumann CR, Werth E, Stocker R, Ludwig S, Bassetti CL. Sleep-wake disturbances 6 months after traumatic brain injury: a prospective study. Brain. 2007;130(7):1873-1883. doi:10.1093/brain/awm109

11. Alhola P, Polo-Kantola P. Sleep deprivation: impact on cognitive performance. Neuropsychiatr Dis Treat. 2007;3(5):553-567.

12. Venter R. Role of sleep in performance and recovery of athletes: a review article. S Afr J Res Sport Phys Educ Recreation. 2012;34 (1):167-184

13. Bramley H, Henson A, Lewis M, Kong L, Stetter C, Silvis M. Sleep disturbance following concussion is a risk factor for a prolonged recovery. Clin Pediatr (Phila). 2017;56(14):1280-1285. doi:10.1177/ 0009922816681603

14. Schwartzberg L, Aslanyan L, Angelo N, et al. Cranial strain patterns associated with concussions. J Am Osteopath Assoc. 2020;120(9): In Press. doi:10.7556/jaoa.2020.098

15. Yao YC, Zwibel H, Angelo N, Leder A, Mancini J. Effectiveness of osteopathic manipulative medicine vs concussion education in treating student athletes with acute concussion symptoms. J Am Osteopath Assoc. 2020;120(9): In Press. doi: 10.7556/ jaoa.2020.099

16. Ettlinger $\mathrm{H}$, Gintis B. Osteopathy in the cranial field. In: DiGiovanna EL, Schiowitz S, Dowling DJ, eds. An Osteopathic Approach to Diagnosis and Treatment. 3rd ed. Philadelphia, PA: Lippincott Williams \& Wilkins; 2005:109-112.

17. King HH. Osteopathic cranial manipulative medicine. In: Seffinger MA, executive ed. Foundations of Osteopathic Medicine. 4th ed. Wolters Kluwer s; 2018:885-905

18. Warren C, Keys J, Pierce-Talsma S. Osteopathic cranial manipulative medicine in the setting of concussion. J Am Osteopath Assoc. 2018;118(6):e41-e42. doi:10.7556/jaoa.2018.088

19. Huard, $Y$. Influence of the venous sinus technique on cranial hemodynamics. In: King HH, ed. Proceedings of International Research Conference: Osteopathy in Pediatrics at the Osteopathic Center for Children in San Diego, CA 2002. Indianapolis, IN: American Academy of Osteopathy; 2005: 32-36.

20. Cutler MJ, Holland BS, Stupski BA, Gamber RG, Smith ML. Cranial manipulation can alter sleep latency and sympathetic nerve activity in humans: a pilot study. J Altern Complement Med. 2005;11(1):103-108. doi:10.1089/acm.2005.11.103

21. Sport Concussion Assessment Tool - 5th edition. Br J Sports Med. 2017;51(11):851-858. doi:10.1136/bjsports-2017-097506SCAT5

22. Daneshvar DH, Nowinski CJ, McKee AC, Cantu RC. The epidemiology of sport-related concussion. Clin Sports Med. 2011;30 (1):1-17. doi:10.1016/j.csm.2010.08.006

23. Marar M, Mcllvain NM, Fields SK, Comstock RD. Epidemiology of concussions among United States high school athletes in 20 sports. Am J Sports Med. 2012;40(4):747-755. doi:10.1177/ 0363546511435626

24. Sarris J, Byrne GJ. A systematic review of insomnia and complementary medicine. Sleep Med Rev. 2011;15(2):99-106. doi:10.1016/j.smrv.2010.04.001

25. Oliveira D, Hachul $H$, Tufik S, Bittencourt L. Effect of massage in postmenopausal women with insomnia: a pilot study. Clinics (Sao Paulo). 2011;66(2):343-346. doi:10.1590/ s1807-59322011000200026 
26. Ko YL, Lee HJ. Randomized controlled trial of the effectiveness of using back massage to improve sleep quality among Taiwanese insomnia postpartum women. Midwifery. 2014;30(1):60-64 doi:10.1016/j.midw.2012.11.005

27. Azad N, Byszewski A, Sarazin FF-A, McLean W, Koziarz P. Hospitalized patients' preference in the treatment of insomnia: pharmacological versus non-pharmacological. Can J Clin Pharmacol. 2003;10(2):89-92.

28. Jaffee MS, Winter WC, Jones CC, Ling G. Sleep disturbances in athletic concussion. Brain Inj. 2015;29(2):221-227. doi:10.3109/ 02699052.2014 .983978

29. Laker SR. Sports-related concussion. Curr Pain Headache Rep. 2015;19(8):41. doi:10.1007/s11916-015-0510-3

30. McCallister A, Brown C, Smith M, Ettlinger H, Baltazar GA. Osteopathic manipulative treatment for somatic dysfunction after acute severe traumatic brain injury. J Am Osteopath Assoc. 2016;116(12): 810-815. doi:10.7556/jaoa.2016.157
31. Patel KG, Sabini RC. Safety of osteopathic cranial manipulative medicine as an adjunct to conventional postconcussion symptom management: a pilot study. J Am Osteopath Assoc. 2018;118 (6):403-409. doi:10.7556/jaoa.2018.061

32. Castillo I, Wolf K, Rakowsky A. Concussions and osteopathic manipulative treatment: an adolescent case presentation. J Am Osteopath Assoc. 2016;116(3):178-181. doi:10.7556/jaoa.2016.034

33. Guernsey DT III, Leder A, Yao S. Resolution of concussion symptoms after osteopathic manipulative treatment: a case report. J Am Osteopath Assoc. 2016;116(3):e13-e17. doi:10.7556/ jaoa.2016.036

34. Burm FV, Goedhart A. Preliminary evidence on the efficacy of cranial osteopathy in professional football. Br J Sports Med. 2017;51 (4):400-401. doi:10.1136/bjsports-2016-097372.296

๑) 2020 American Osteopathic Association

\section{JAOA Submissions: Online-Only Content}

Videos and slides can be great supplemental components to published research. The Journal of the American Osteopathic Association encourages authors to include such online-only content with their manuscript submissions. Contact the JAOA's editorial assistant at jaoa@osteopathic.org for more information. 